\title{
Transformaciones espacio-temporales de la cobertura vegetal en el Parque Nacional Corcovado, 1960-2014
}

\author{
Carlos Morera B. ${ }^{1}$, Luis Fernando Sandoval M. ${ }^{1} \&$ Josep Pintó ${ }^{2}$
}

1. Profesor, Escuela de Ciencias Geográficas, UNA. Apdo. postal 86-3000. Heredia, Costa Rica; cmorera@una.cr, luifersandoval@gmail.com

2. Profesor, Laboratorio de Análisis y Gestión del Paisaje (LAGP), Universidad de Girona, Pl. Ferrater Mora, 1-17071_Girona, España; josep.pinto@udg.edu

Recibido 01-VIII-2017. Corregido 27-X-2017. Aceptado 25-XI-2017.

\begin{abstract}
Space-time transformations of vegetation coverage in Corcovado National Park, 1960-2014. Regional studies evaluating spatial-temporal transformations of vegetation in Costa Rica, especially within National Parks, are scarce. Therefore, this paper analyses the vegetation distribution during 1960, 1976, 1997 and 2014 in Corcovado National Park. This protected area is located in the Osa Peninsula, Costa Rica, and represents the Northern most tropical rain forest on the Pacific coast of America. This area offers a great wealth of biodiversity due to its geological formation, isolation for long time periods, and its particular climatic conditions that generate unique ecosystems such as cloud forests associated with ocean situated close to hill breezes located over 500 masl, as well as dense tropical forest. This study evaluates the spatial distribution of vegetation based on maps that resulted from the process of photo-interpretation of 1960, 1976, 1997 and 2012, as well as from the landscape index analysis. It concludes that during the study period, the vegetation changes have been minimal. Instead, in the few areas impacted by human activity (small-scale agriculture and pasture lands) an ecological restoration has occurred during recent decades. In addition, this research suggests that the recovering forest cover within the park and even within the Osa Peninsula has been expanding the cloud forest. An increase and contraction relationship between the different categories (Cloud forest and forests as well of flooded forest and forest in flat zones) was found. Furthermore, this study suggests the need of permanent plots in order to monitor vegetation and identify the factors that explain this previous process. Rev. Biol. Trop. 66(1): 352-367. Epub 2018 March 01.
\end{abstract}

Key words: Tropical rain forests; vegetation mapping; Corcovado National Park; monitoring land-use; vegetation categories.

Durante las últimas décadas, la comprensión de los efectos humanos sobre el ambiente ha sido prioridad en temas de investigaciones (Foster, Orwlg, \& McLachlan, 1996), y este interés se fundamenta en el incremento del impacto antrópico sobre los procesos ecológicos de los últimos dos siglos (Manies \& Mladenoff, 2000). El crecimiento de la población, así como el desarrollo industrial han generado el surgimiento de una nueva era geológica conocida como el antropoceno, con efectos de la actividad antrópica sobre los ciclos biogeoquímicos del planeta (Morera, 2015). De esta forma, el efecto del cambio climático implica transformaciones ecológicas como son: expansión y contracción de la distribución de ecosistemas, fragmentación de los ecosistemas naturales, extinción de algunas especies y proliferación de otras (Young, 2014), lo cual se evidencia en la distribución espacial de la cobertura vegetal (Palacio-Aponte, 2014).

De acuerdo con Geist, McConnell, Lambin, Moran, Alves y Rudel (2006), la cobertura vegetal es el resultado de factores antropogénicos y biofísicos, que se combinan para su transformación. Por ejemplo, una investigación de la distribución de la vegetación nativa en Monte Árido Central de Argentina, encontró 
que las principales diferencias en los cambios de la vegetación se deben a la presencia de procesos geomorfológicos activos (Flores, Suvires, \& Dalmasso, 2015). Los estudios que abordan las transformaciones de la vegetación dentro de espacios protegidos, como la realizada por Aldana y Bosque (2008) en el Parque Nacional Sierra de la Culata, Venezuela, durante 1988 - 2003, así como el estudio de Domínguez (2011) en sector sur del Parque Nacional Natural los Katíos, Colombia entre 1989-2010, presentan periodos menores de 25 años. Igual sucede con el caso de la investigación realizada por Velázquez et al. (2003) en Oaxaca, México, que analiza los procesos de cambios en la cobertura durante 20 años. Otro estudio sobre la temática es el realizado por Sahagún, Reyes, Flores y Vargas (2011) en la Sierra Madre Oriental del estado de San Luis Potosí, México, quienes modelan el cambio en el uso de suelo y la vegetación, basados en imágenes de satélite para un periodo de 16 años (1989 - 2005). Los anteriores estudios se enmarcan en períodos inferiores a 25 años, que en muchos casos no evidencian las transformaciones espaciales de la vegetación de relevancia como pueden ser estadios de regeneración.

En Costa Rica, el estudio de la distribución de la vegetación se remonta a mediados del siglo XIX, cuando Anders S. Oersted realiza investigaciones pioneras; y posteriormente, en la segunda mitad del siglo XIX, los estudios de: Moritz Wagner, Kart Scherzer, Karl Hoffman, Herman Wenland, Felix Bally, Henry Pittier, Adolfo Tonduz y Pablo Bioley como lo plantea Hilje (2013). Dentro de este periodo, destacan los esfuerzos de Helmut Polakowski por cartografiar la fitogeografía del país, así como los realizados por Merker al efectuar una descripción de las formaciones vegetales de Costa Rica (Vargas, 2009). Durante las últimas décadas, con el desarrollo de las tecnologías espaciales han proliferado las investigaciones que abordan la distribución espacial de la vegetación, como las realizadas por: Veldkamp y Fresco (1996); Calvo et al. (1999); Sánchez, Foley, Hamilton, Calvo, Arroyo y Jiménez (2002); Calvo y Ortiz (2011); Morera (2010); y Morera y Sandoval
(2012, 2013, 2015). Además, destacan los estudios realizados por el Sistema Nacional de Áreas de Conservación (SINAC, 1996; 2007) sobre el análisis de vacíos de conservación en Costa Rica, que cartografían los ecosistemas del país. En el caso específico de la Península de Osa, donde se ubica el área de estudio seleccionada, que es el Parque Nacional Corcovado, a principios de este siglo, se realizó un mapa de los ecosistemas de la Área de Conservación Osa (Kappele, Castro, Acevedo, González, \& Monge, 2002).

A pesar de este aumento de investigaciones, que abordan la cartografía de la vegetación, las mismas se concentran en un año, o son de periodos menores de 25 años que no permiten identificar adecuadamente las transformaciones, debido a que los cambios en la distribución espacial de la cobertura de vegetación suceden durante escalas temporales mayores de 25 años (Green, Schweik, \& Randolph, 2005). Con base en la premisa anterior, este artículo, analiza la distribución espacial de la cobertura vegetal durante los últimos 54 años en el área que actualmente ocupa el Parque Nacional Corcovado, con el propósito de identificar las transformaciones espacio-temporales que, como abordaje innovador para escenarios tropicales, permite monitorear dinámicas de vegetación para periodos mayores a los estudiados hasta la actualidad, lo cual permitiría identificar acciones que potencien la conservación de estos ecosistemas, fundamentales para la biodiversidad del país. Así, esta investigación recurre a la fotointerpretación para construir una serie temporal que permite analizar la distribución de la cobertura vegetal a lo largo de 54 años (1960 - 2014), para lo cual se aplicó un abordaje paisajístico de índices biométricos y una matriz de cambios para facilitar el análisis de las transformaciones espaciales de la cobertura natural.

\section{MATERIALES Y MÉTODOS}

Área de Estudio: El Parque Nacional Corcovado se localiza entre las coordenadas geográficas $\left(8^{\circ} 25^{\prime} 55^{\prime \prime}-8^{\circ} 44^{\prime} 00^{\prime \prime} \mathrm{N}-83^{\circ} 24^{\prime} 50^{\prime}\right.$ 


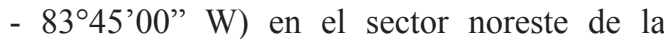
Península de Osa (Fig. 1). Durante el Plioceno Medio, diversas cuencas sedimentarias fueron rellenadas con sedimentos fosilíferos provenientes del Complejo de Nicoya, que es la unidad litológica más antigua datada en Costa Rica y que aflora en las penínsulas de Osa y Nicoya (Denyer y Kussmaul, 2000). Además, parte del área de estudio está formada por relleno aluvial y montañas que generan un gradiente ecológico con altitudes de más de $500 \mathrm{~m}$, como los cerros Mueller, Brujo y Rincón, en lugares relativamente cercanos a la costa $(6 \mathrm{~km})$.

La cobertura natural dominó los espacios, que actualmente forman parte del Parque Nacional Corcovado, hasta finales de la década de los sesenta y principios de los setenta en que se inicia su colonización. Aunque en general, los suelos de la Península de Osa son ultisoles e inceptisoles, con capacidad de uso básicamente forestal, debido a las altas precipitaciones y las fuertes pendientes que dominan en el área, e incluso los suelos en áreas planas, como los que se presentan en la cuenca del río Sirena, muestran restricciones agrícolas por sus limitaciones con el drenado (Lewis, 1983).

El 24 de octubre de 1975, se estableció el Parque Nacional Corcovado, según el Decreto $\mathrm{N}^{\circ} 53-57^{\mathrm{a}}$ con una extensión de 34346 ha (Vaughan, 1981) para proteger la biodiversidad del área de estudio, que estaba amenazada por la expansión de la frontera agrícola. El parque fue ampliado con el Decreto $\mathrm{N}^{\circ} 11148 \mathrm{~A}$ del 5 de febrero de 1980 y ratificado con la Ley $\mathrm{N}^{\circ} 6794$ del 25 de agosto de 1982. Luego de su establecimiento, el área de estudio ha sido motivo de diversas investigaciones, especialmente, sobre el hábitat de felinos, pioneras a nivel de ecosistemas neotropicales (Carrillo, 2000). En 1997, de acuerdo con Sánchez, Benoit, Calvo y Moorthy (2002), el Parque Nacional Corcovado protegía el $45 \%$ de los bosques de la península de Osa. Asimismo, el ecoturismo se ha transformado, siendo una de

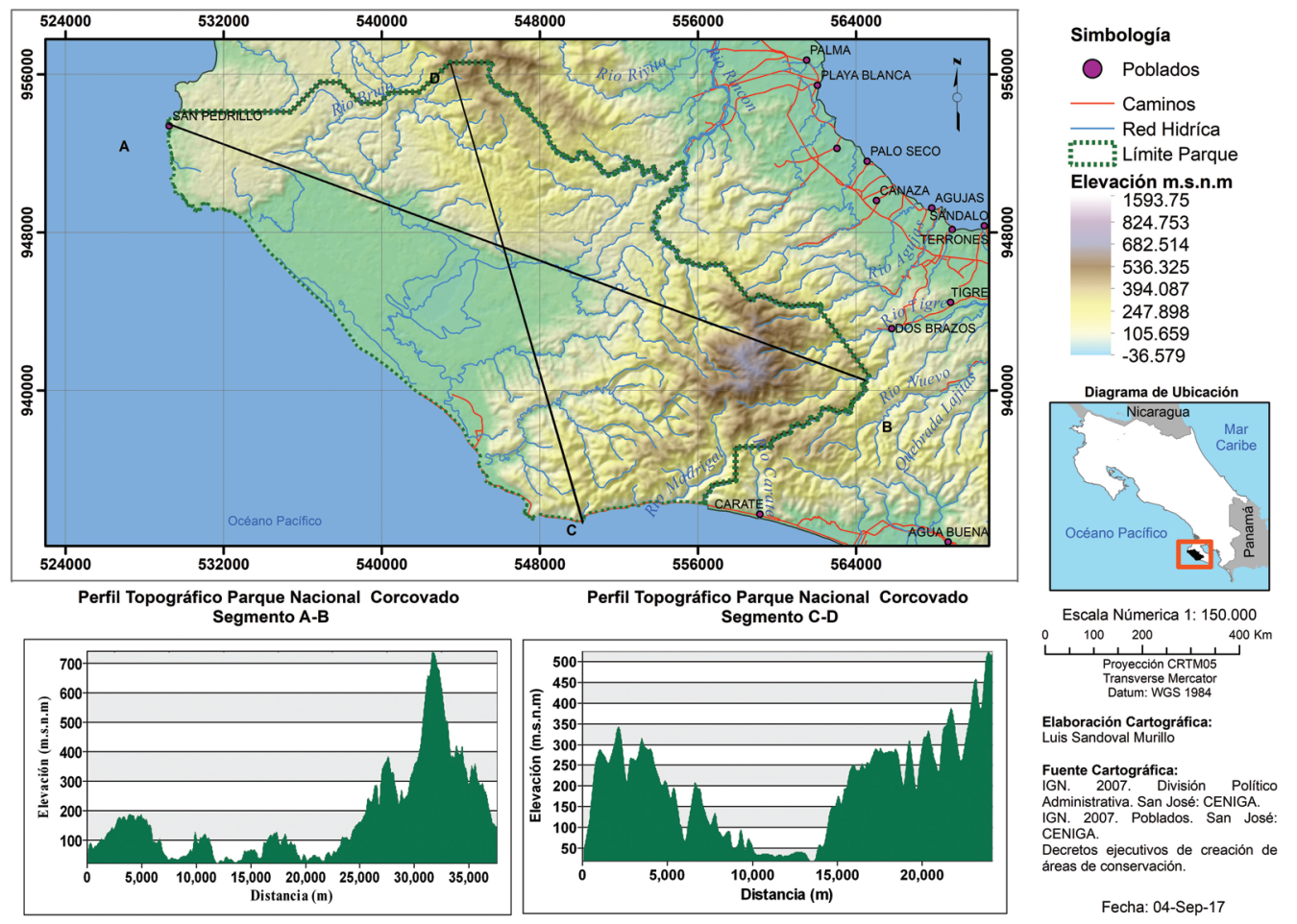

Fig. 1. Localización del Parque Nacional Corcovado.

Fig. 1. Corcovado National Park location. 
las principales actividades económicas de la Península de Osa, siendo el principal atractivo la observación de fauna dentro del Parque Nacional Corcovado (Morera, 2001).

Identificación de los periodos de estudio y las categorías de vegetación: Para la realización de esta investigación, primeramente, se seleccionaron las fotografías e imágenes satelitales disponibles, como se muestra en el Cuadro 1, el cual inicia con el año 1960, porque, aunque existen otros proyectos de fechas anteriores, las escalas son demasiado generales, no permitiendo reconocer transformaciones en la distribución espacial de la vegetación acordes con el objetivo de esta investigación. De acuerdo a la literatura consultada, no se han realizado estudios que aborden las transformaciones espaciales en las coberturas vegetales por periodos mayores a 30 años a nivel de paisaje (Nagendra, Southworthm, \& Tucker, 2003). Sin embargo, los cambios tecnológicos de los últimos años, han generado fuentes de información diferentes, que requieren complementarse cuando se evalúan transformaciones en periodos de más de 50 años, como es el caso de la presente investigación. Se han elaborado estudios como el implementado por PalacioAponte (2014) en la zona costero-palustre de
Campeche, México, para periodos de 28 años, basado en imágenes Landsat; sin embargo, este tipo de información que inicia en 1972 con el Landsat 1, no está disponible para el área de estudio, por lo cual se recurrió a otra fuente como son las fotografías aéreas, y se combinaron los métodos

Las fotografías aéreas e imágenes corresponden a los años: 1960, 1976, 1997 y 2012 con escalas entre 1:60000 y 1:35000. Durante la digitalización, por medio de la fotointerpretación, se consideró como unidad mínima a representar cartográficamente un área específica para cada uno de los años considerados, para lo cual se utilizó la siguiente fórmula:

$$
\mathrm{UMC}=(\operatorname{Lpv} \times \mathrm{E} \times 3)^{2}
$$

Donde:

UMC: Unidad Mínima Cartografiable, expresada en $\mathrm{m}^{2}$.

Lpv: Límite de percepción visual $(0.2 \mathrm{~mm}$ a escala del plano) pero expresado en $\mathrm{m}$.

E: Escala del mapa.

Así, la unidad mínima cartografiable fue de $1296 \mathrm{~m}$ en 1970, 576 y $441 \mathrm{~m}$ en 1976, $441 \mathrm{~m}$ en 1997 y 225 en 2012. Los periodos considerados para el análisis comparativo de cambios en el paisaje fue de 16 años para 1960 - 1976,

CUADRO 1

Información de Fotografías Aéreas e Imágenes utilizadas en esta investigación

TABLE 1

Information of aerial photographs and images used in this investigation

\begin{tabular}{|c|c|c|c|c|c|c|c|}
\hline Año & Proyecto & Rollo & Línea & \# de Fotos & Escala & Fecha & $\begin{array}{c}\text { Unidad Mínima } \\
\text { Cartografiable }(\mathrm{m})\end{array}$ \\
\hline \multirow[t]{3}{*}{1960} & 55AM73 & 104 & $91 \mathrm{~A}$ & $972,973,974,975$ & $1: 60000$ & 14/01/1960. & 1296 \\
\hline & & 104 & 90 & $\begin{array}{c}956,957,958,959,960 \\
961,962,963,964\end{array}$ & $1: 60000$ & 14/01/1960. & \\
\hline & & 104 & 89 & 1056,1057 & $1: 60000$ & 14/01/1960. & \\
\hline \multirow[t]{2}{*}{1976} & Corcovado & 103 & 287 & $\begin{array}{c}5851,5852,5853,5854, \\
5855,5856,5857,5858,5859\end{array}$ & $1: 40000$ & 06/02/1976 & 576 \\
\hline & & 103 & 279 & $\begin{array}{l}5875,5876,5877,5878 \\
5879,5880,5881,5882\end{array}$ & $1: 40000$ & 06/02/1976 & \\
\hline 1978 & Madrigal-Carate & 129 & 270 & $\begin{array}{c}12054,12055,12056 \\
12057,12058,12059,12060\end{array}$ & $1: 35000$ & 09/01/1978. & 441 \\
\hline 1997 & Terra 1997 & N/A & N/A & N/A & $1: 40.000$ & 01/01/1997 & 576 \\
\hline 2012 & Rapidaye & N/A & N/A & N/A & $1: 25.000$ & $30 / 08 / 2012$ & 225 \\
\hline
\end{tabular}


mientras que para 1976 - 1997 fue de 11 años y para 1997 - 2014, de 16 años con una diferencia de cinco años. Para la realización de este proyecto, se fotointerpretaron las imágenes aéreas y de satélite con un estereoscopio de espejos (Adan Gordon Enterprise INC, Type F-71). Aunque para el análisis de la información de 2014 se podía aplicar una clasificación digital, con el objetivo de reducir el sesgo, se utilizó la misma metodología para los diferentes años. Dos de los autores realizaron la fotointerpretación, uno fue responsable de la identificación y otro se encargó de supervisar, basado en su conocimiento del área de estudio y en la información de campo. En el caso del periodo 1976, una pequeña parte requirió imágenes de 1978, ya que ninguno de los dos años cubría el área de interés en totalidad. Con el objetivo de evaluar la veracidad de la información del mapa de cobertura vegetal de 2012 se actualizó en el campo durante el 2014, por lo cual se utiliza este último año para el análisis. Además, se aplicó una matriz de confusión (Chuvieco, 2010) para esto se estableció una red de 260 puntos dentro del área de estudio. Basado en lo anterior, durante el mes de octubre de 2013 y de 2014, se evaluó la coincidencia de lo fotointerpretado con el uso real, para lo cual se tomaron 78 puntos $(30 \%)$ al azar que fueron verificados en visitas de campo por medio de la lectura de un sistema de posicionamiento global (GPSMP- Serie 78), que presentó una confianza de un $92 \%$ (Cuadro 2).
De acuerdo al trabajo de campo y la fotointerpretación; así como la revisión bibliográfica, se identificaron seis categorías de coberturas vegetales según su fisonomía (Cuadro 4). Para analizar la distribución de las mismas, se utilizó el programa Patch Analyst, una extensión del programa Arc-GIS que calcula índices paisajísticos. Para el análisis se seleccionaron los índices: superficie de cada categoría y número de fragmentos, por ser los más relevantes con el objetivo de esta investigación, debido a la extensión de este artículo. Asimismo, se realizó una matriz de cambios de las coberturas para apoyar el estudio.

\section{RESULTADOS}

Las categorías de las coberturas vegetales fueron jerarquizadas de acuerdo con las especies identificadas en el trabajo de campo y el apoyo bibliográfico (Cuadro 3), estas fueron aplicadas en la elaboración de los mapas para el año 1960, 1976, 1997 y 2014 (Cuadro 5, Fig. 2 y Fig. 3). Además, se realizaron mapas de los cambios presentados durante los periodos: 1960 - 1976, 1976 - 1997 y 1997 - 2014 (Fig. 4 y Fig. 5). Las superficies de las diferentes categorías analizadas, en general, no muestran grandes diferencias, lo cual se relaciona con el establecimiento del Parque Nacional Corcovado en el año 1975, cuando se preservaba la mayoría de la cobertura natural. El área de estudio presentaba una predominancia de

CUADRO 2

Matriz de Confusión

TABLE 2

Confusion matrix

\begin{tabular}{lccc}
\multicolumn{1}{c}{ Categoría } & Puntos Muestreados & Puntos que coincidieron & \% de coincidencia \\
Bosque nuboso & 8 & 7 & 87.5 \\
Bosque & 45 & 42 & 93.3 \\
Bosque inundado & 6 & 6 & 100.0 \\
Vegetación costera & 5 & 4 & 80.0 \\
Vegetación lacustre & 8 & 8 & 100.0 \\
Actividad antrópica & 6 & 6 & 100.0 \\
Total & 78 & 73 & 93.6 \\
\hline
\end{tabular}




\section{CUADRO 3}

Parque Nacional Corcovado. Principales especies por cobertura vegetal

TABLE 3

Corcovado National Park. Main species by vegetation cover

Tipo de vegetación

Bosque Nuboso

Bosque

Bosque Inundado

Vegetación Costera

Vegetación lacustre

Actividad antrópica

\section{Especies Identificadas}

Presencia de los robles como el Quercus insignis y Quercus rapurahuenis, así como helechos arborescentes (Cyathea trichiata) en el sotobosque Bognounou F. et al. (2011) además, la presencia de Alfaroa guanacastensis. Oreomunnea pterocarpa (Gavilan) y Ticodendron inconitum (Jaul Macho).

Se localizan especies como cedro amargo (Carapa guianensis), fruta dorada (Virola sp), sangrillo (Pterocarpus officinalis), guayabon (Terminalia lucida), mientras en las áreas de pendiente fuerte y onduladas con presencia de especies como ceiba (Ceiba Pentandra) y Sloanea laurifolia.

Está dominada por la palma de yolillo (Raphia Taedigera), por lo que algunos autores lo denominan Yolillales, que produce un coyol que es alimento de animales silvestres como el saíno (Dicotyles tacaju), chancho montes (Dicotyles pecari), tepezquistles (Cunuculus paca), danta (Tapirus bairdii).\}, lo cual es fundamental para el mantenimiento de las grandes poblaciones de estas especies dentro del Parque Nacional.

Dominan Rhizophora mangle (mangle rojo). Avicennia nítida (Mangle negro). Mora oleira (Alcornoque de la costa Pacífica o Chaperno de Suampo) y algunas especies naturalizadas como Hibiscus Tiliaceus. Además, se identificaron algunos árboles dominando en el dosel como el ceibo (Ceiba Pentandra) y el zapatón (Pachira acuática).

Se reconocieron en las visitas de campo el Pennisetum sp, que se asocia con algunas especies de menor presencia como chile perro (Poligonum s). Aeschynomene sp. y Ludwigia.

Presencia de granos básicos como el maíz (Zeas mays) y los frijoles (Phaseolus vulgaris). Asimismo, se incluyen las gramíneas utilizada para el pastoreo, las cuales no se logró comprobar en el trabajo de campo.

Fuente: elaboración propia a partir de las visitas de campo apoyada por (Vaughan. 1981) y (Quesada, Jiménez, Zamora, Aguilar, \& González, 1999).

vegetación natural del $99.8 \%$, lo que provocó que la actividad antrópica existente se constituyera en áreas de restauración para el 2014. Durante 1960 y 1976, las actividades antrópicas mostraban una localización muy puntual en los sectores planos de la cuenca del Río Sirena, la cual se incrementó pasando de un $0.02 \%$ en 1960 , a un $1.80 \%$ en 1976 , lo que evidencia la expansión de la frontera agrícola durante este periodo, por la expansión de la ganadería y el cultivo de los granos básicos (Frijol, arroz y maíz).

El bosque nuboso se localiza en las cimas mayores de $550 \mathrm{~m}$ como en los cerros Rincón (745 m) y Mueller (744 m), localizados en el sector Sur-este del Parque Nacional; así como en el sector noreste en el cerro Brujo (617 m) con fuerte tendencia a expandirse, reduciendo el número de fragmentos y aumentando la conexión de los mismos. El bosque nuboso representó un $11.1 \%$ en 1960 y $11.5 \%$ en 1976, mientras que para 1997 fue de $13.7 \%$ y en 2014 de $21.2 \%$ del área de estudio. El bosque ocupó el $85.6 \%$ en 1960, el $83.1 \%$ en 1976, el $83.3 \%$ en 1997 y el $72.1 \%$ en 2013 .

En cuanto al número de fragmentos de las coberturas (Fig. 6), se identificaron en el bosque nuboso cinco para 1960 y 2014, 12 en 1978 y nueve en 1997. La categoría de bosque presentó 14 fragmentos tanto en 1960 como en 1976, 10 en 1997; mientras que en el bosque inundado se reconoció solamente un fragmento y en la vegetación lacustre dos en 1960, uno en 1976 y 1997 y cuatro en 2014. La vegetación 


\section{Cambios entre 1960 y 1976}

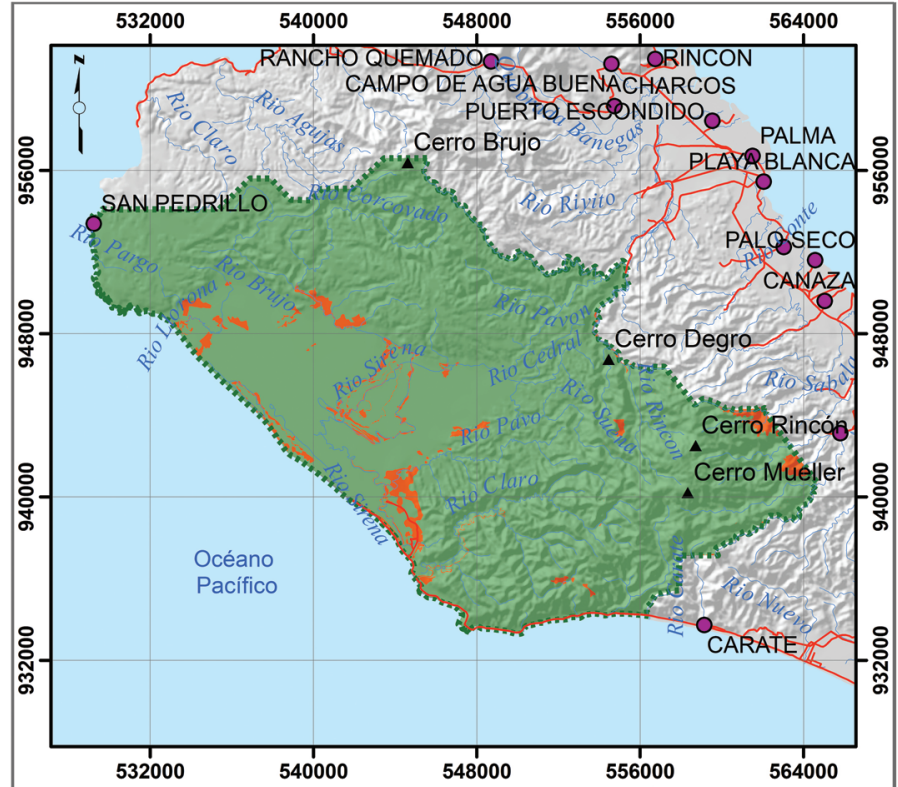

\section{Cambios entre 1976 y 1997}

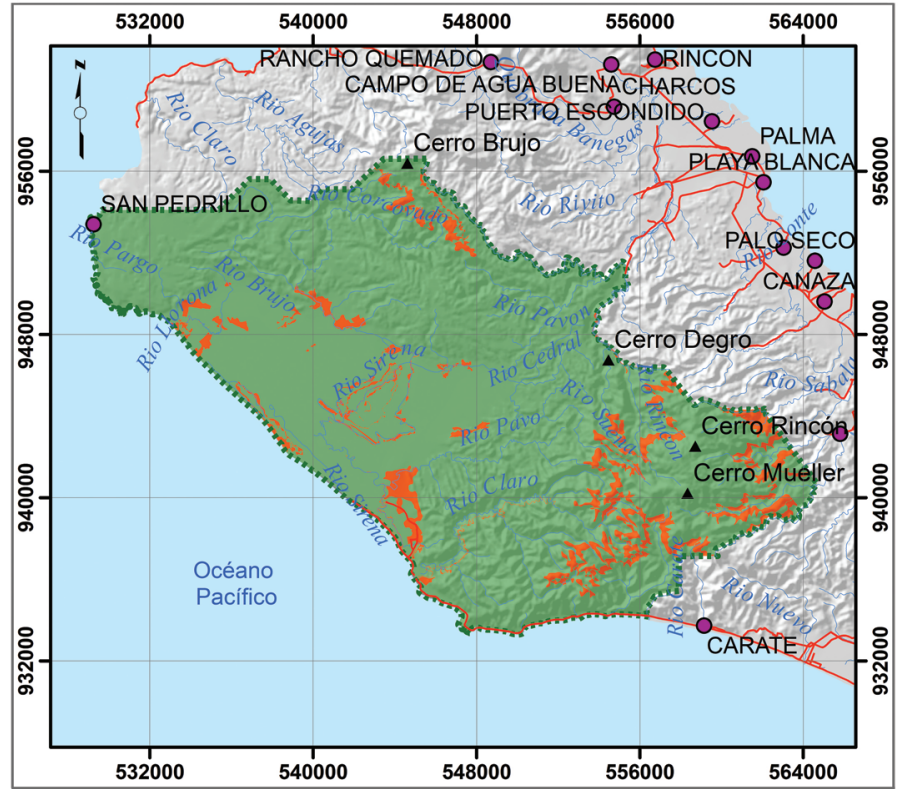

\section{Simbología}

- Poblados

- Cerros

$\frown$ Caminos

$\sim \sim$ Red Hidríca

21:" Límite Parque

Cambios de Cobertura

clasificación

Cambio

No cambio

Diagrama de Ubicación

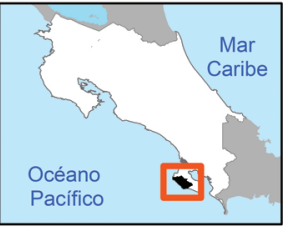

Escala Númerica 1: 300.000 0 $100200 \quad 400 \mathrm{Km}$

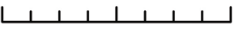

Proyección CRTM05 Transverse Mercator Datum: WGS 1984

SIG y Cartográfia:

Luis Sandoval Murillo

\section{Fuente Cartográfica:}

IGN. 2007. División Político Administrativa. San José: CENIGA.

IGN. 2007. Poblados. San José: CENIGA.

Decretos ejecutivos de creación de áreas de conservación.

Fuente Fotogramétrica:

55AM73.

1960.

Fotografias aéreas

Proyecto 55AM73.

Escala 1: 60.000 .

Corcovado. 1976

Fotografias aéreas

Proyecto Corcovado.

Escala 1: 40.000

Terra. 1997. Fotografias aéreas Proyecto Terra.

Escala 1: 40.000 .

Fecha: 18-Aug-17

Fig. 2. Cobertura Vegetal, Parque Nacional Corcovado, 1960 y 1976.

Fig. 2. Vegetation cover, Corcovado National Park, 1960 and 1976. 


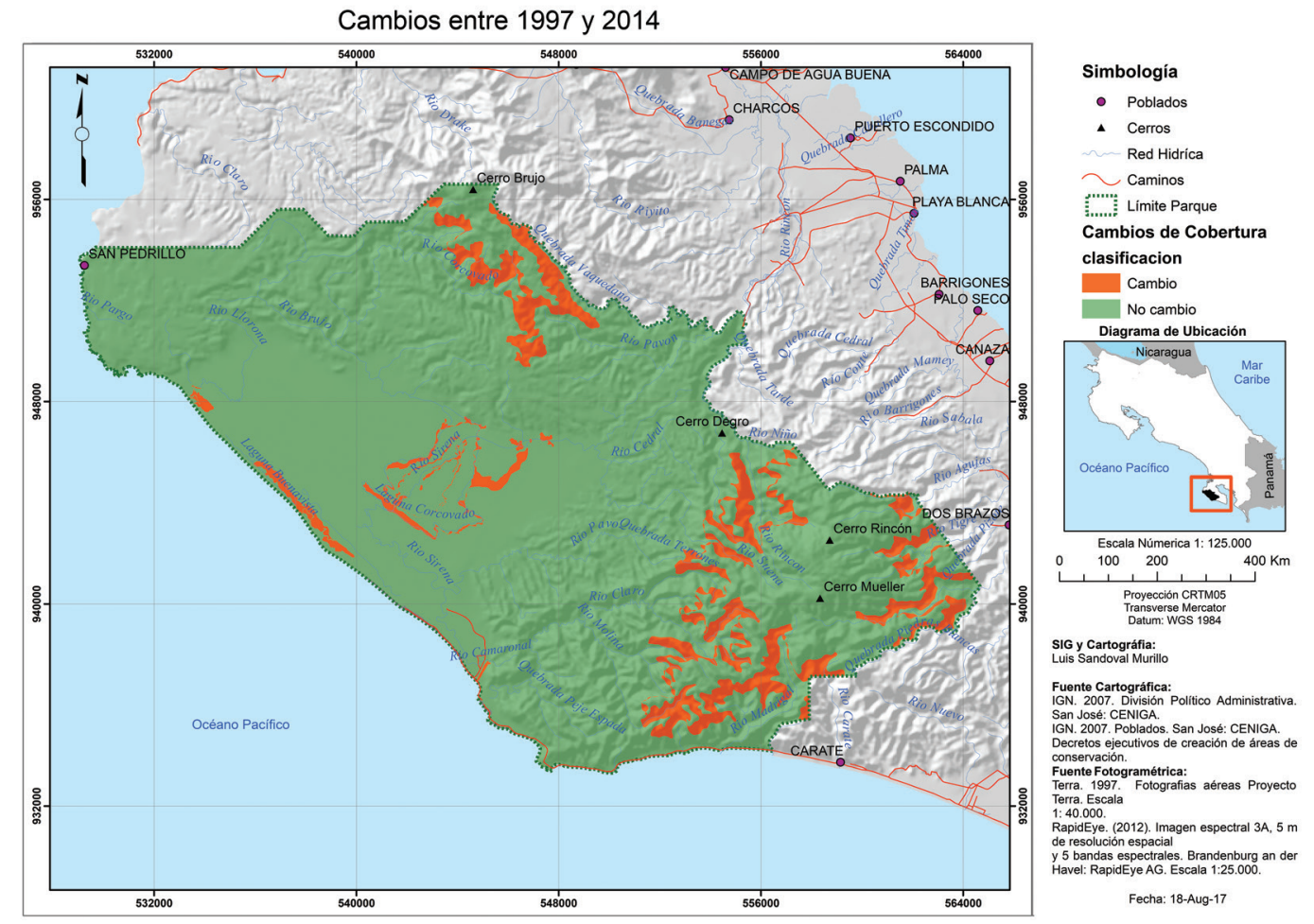

Fig. 3. Cobertura Vegetal. Parque Nacional Corcovado. 1997 y 2014.

Fig. 3. Vegetation cover, Corcovado National Park, 1997 and 2014.

costera presentó dos fragmentos en 1960, 1976 y 1997 y cuatro en 2014; y la actividad antrópica constituyó 11 en 1960, seis en 1997, cuatro en 1997 y uno en 2014. Los cuerpos de agua fueron cinco en 1960, nueve en 1998 y 2014; y cuatro en 1997. Respecto a los cambios en las superficies de las coberturas naturales, durante el periodo 1960 - 1976 el $92.0 \%$ de la cobertura se mantuvo bajo la misma categoría, mientras, durante los años 1976 - 1997 el 87.7 \% presentó la misma condición y en el lapso 1997 - 2014 fue de $86.7 \%$. En cuanto a actividad antrópica, la cual se relaciona con los cultivos de granos básicos y los pastizales para la ganadería extensiva, se presentó un crecimiento vertiginoso entre 1960-1976 de $90 \%$, sin embargo, después del establecimiento del Parque se produce una reducción de estas coberturas, siendo de $95 \%$ durante $1976-1997$ y de $18 \%$ en el periodo 1997-2014. La actividad antrópica estuvo motivada por el establecimiento de tierras para agricultura y ganadería, y generó la destrucción de la cobertura natural, especialmente en los espacios planos alrededor de los ríos Sirena y Claro.

\section{DISCUSIÓN}

En el trabajo de campo se comprobó que dentro del área de estudio en distancias de aproximadamente 8 y $27 \mathrm{~km}$, se presentan transformaciones drásticas de vegetación, relacionadas con el gradiente ecológico que genera cambios de altitud de más de $700 \mathrm{~m}$, lo cual fue reconocido por Willie (1987) en la década de los setentas. Las condiciones anteriormente descritas, aunadas al aislamiento, así como la antigüedad de los basamentos geológicos localizados en los territorios que ocupan actualmente la Península de Osa, y en particular, el Parque Nacional, han generado las circunstancias para la presencia de endemismos, lo cual 


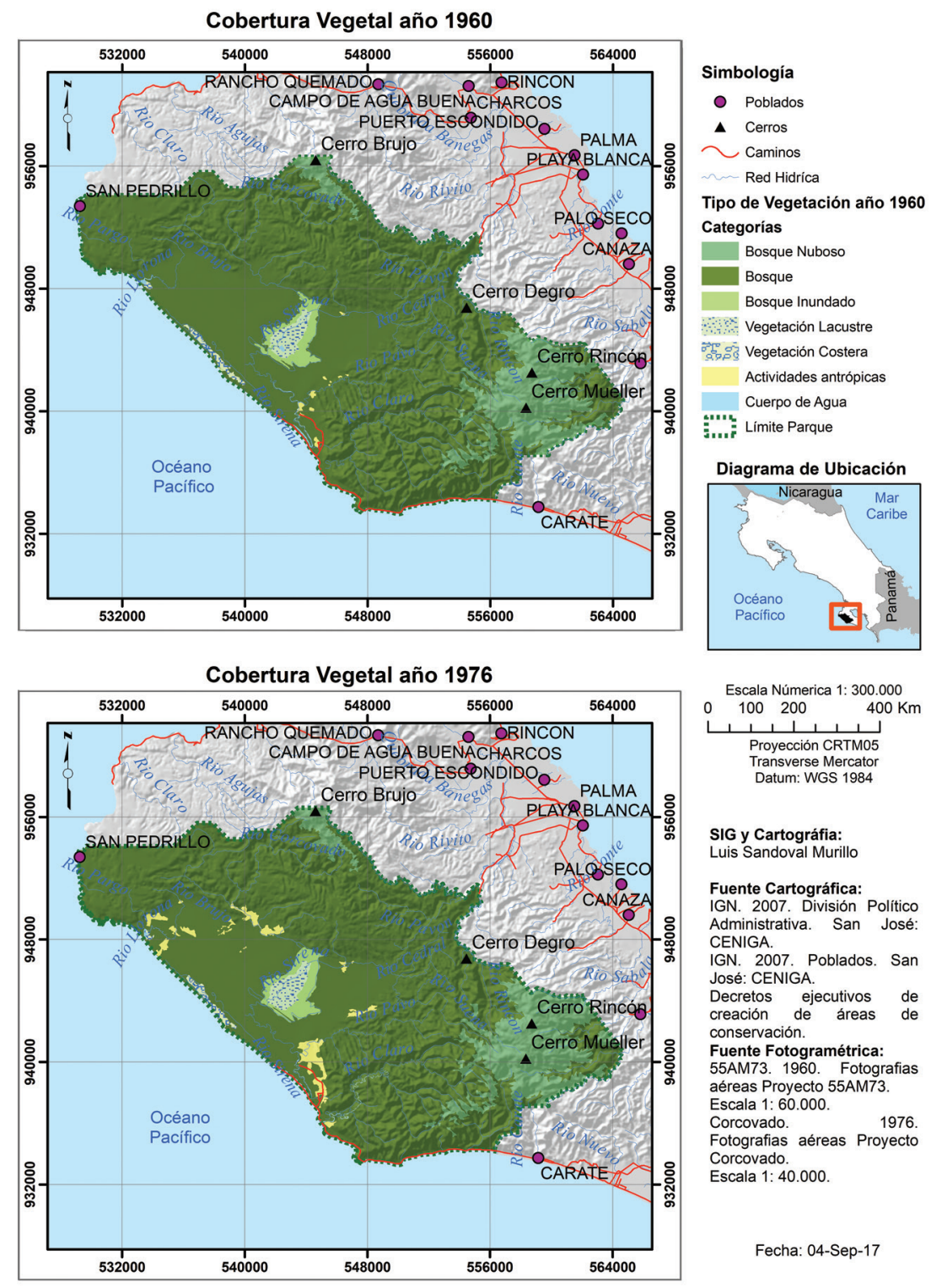

Fig. 4. Cambios en la cobertura vegetal del Parque Nacional Corcovado en el período de estudio1960-1976 y 1976-1997. Fig. 4. Vegetation cover changes of the Corcovado National Park in the study period 1960-1976 and 1976-1997.

se evidencia en las constantes nuevas especies identificadas: el árbol Tapirira lepidota (Hammel, Grayum, \& Aguilar, 2014), la liana Schnella bahiachalensis (Zamora, 2013) y el árbol Couepia osaensis (Santamaría-Aguilar \& Lagomarsino, 2015). El Parque tiene una forma de escenario abierto al océano, con las mayores altitudes localizadas cerca de sus límites, donde se presentan la mayor humedad y la nubosidad originada en el océano y transportada por la brisa marina, concibiendo bosques nubosos muy cerca de la costa y a una baja altitud, como lo afirman Gómez (1986) y Vargas (2009). 


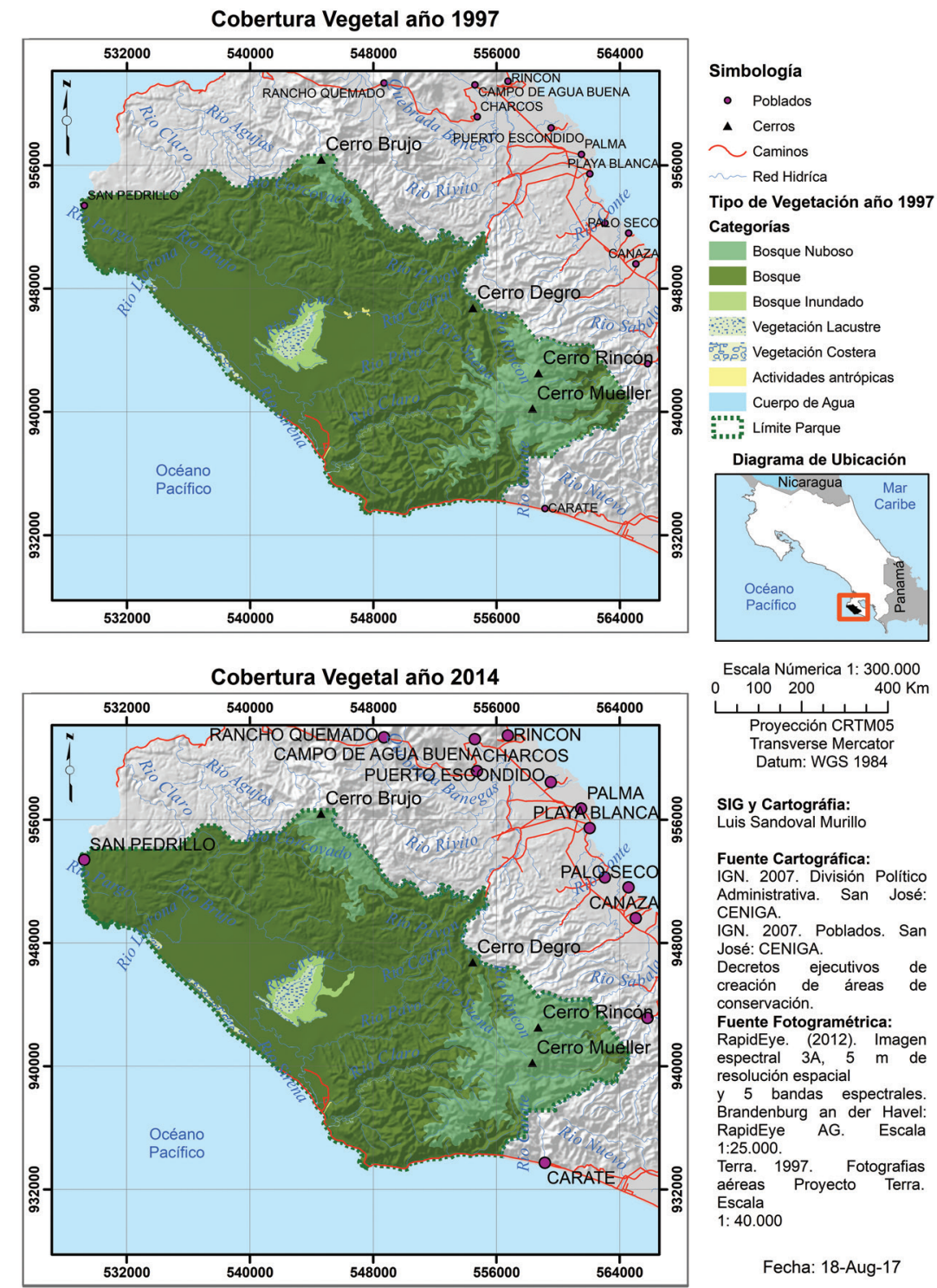

Fig. 5. Cambios en la cobertura vegetal del Parque Nacional Corcovado en el período de estudio 1997-2014.

Fig. 5. Changes in the vegetation cover of the Corcovado National Park in the study 1997-2014.

Alrededor de 706 ha de bosque se transformaron en espacios agropecuarios en el periodo 1960-1976, mientras que en 1976-1997 fueron 23 ha y en 1997-2014 este proceso no ocurrió. El área de estudio mostró una reducción y un aumento de la cobertura boscosa (Bosque nuboso, bosque y bosque inundado), presentando una reducción de $2.3 \%$ en el periodo
1960-1976, mientras que durante 1976-1997, posterior al establecimiento del Parque Nacional, se recuperó la misma cifra eliminada en el periodo anterior (2.3\%), y entre 1997-2014 la reducción fue mínima $(0.2 \%)$.

Un área importante de los bosques se convirtió en bosque nuboso, durante el periodo 1960-1976 de 158.4 ha, mientras en 1976-1997 


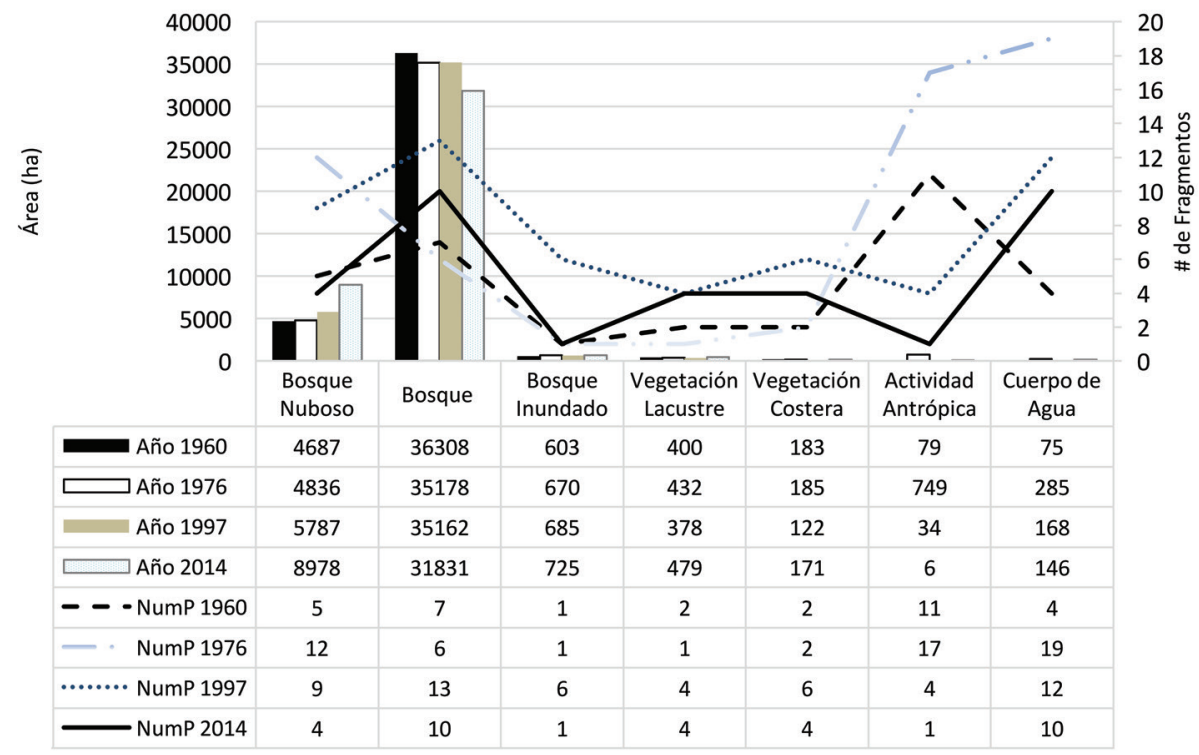

Fig. 6. Superficie y número de fragmentos por Coberturas vegetal. Parque Nacional Corcovado 1960-2014

Fig. 6. Surface and number of fragments by plant covers. Corcovado National Park 1960-2014

\section{CUADRO 4}

Parque Nacional Corcovado. Categorías de la Cobertura vegetal

TABLE 4

Corcovado National Park. Vegetation cover categories

Categorías

Bosque Nuboso

Bosque

Bosque Inundado

Vegetación Costera

Vegetación lacustre

Actividad antrópica

\section{Características}

Es una vegetación determinada por la niebla marina, relacionado con la presencia de serranías cerca de la línea costera que de acuerdo con Gómez (1986), se forman únicamente en la Península de Osa y en el Cerro Iglesias de la Isla del Cocos. En áreas mayores de $500 \mathrm{msnm}$ se identifica la presencia de brisa marina, que genera una nubosidad casi permanente sobre los cerros localizados, donde se incrementa la presencia de epifitas (Quesada, Jiménez, Zamora, Aguilar y González, 1999).

Son bosques tanto maduros como intervenidos que dominan dentro del Parque Nacional. La fotointerpretación; así como el trabajo de campo facilitó categorizar esta vegetación en dos tipos; la localizada en áreas planas y la que se distribuye sobre espacios de fuerte pendiente y onduladas.

Corresponde a las áreas planas alrededor de la laguna Sirena y del cauce del río del mismo nombre, que los suelos se mantienen saturados varios meses durante la época lluviosa (enero-abril). De acuerdo con Gómez (1986) en el país, este tipo de vegetación además se presenta en Barra de Colorado y Tortuguero, similar a lo que se conoce como bosque igapoide localizado en la cuenca del Río Amazonas.

Se refiere a las asociaciones localizadas en áreas costeras que presentan una alta tolerancia al agua salada, así como cerca de los cauces de los ríos.

Se localiza sobre la laguna Sirena, que las altas precipitaciones y las fuertes pendientes han generado un acelerado proceso de colmatación.

Se refiere a los espacios de intervención antrópica para realizar cultivos temporales y ganadería. 
CUADRO 5

Cambios en la cobertura vegetal del Parque Nacional Corcovado en el periódo de estudio

TABLE 5

Changes in the vegetation cover of Corcovado National Park during the study period

Cambio de uso año 1960-1976 (ha)

\begin{tabular}{|c|c|c|c|c|c|c|c|}
\hline \multicolumn{8}{|c|}{ Cobertura 1976} \\
\hline Cobertura 1960 & $\begin{array}{l}\text { Bosque } \\
\text { nuboso }\end{array}$ & Bosque & $\begin{array}{c}\text { Bosque } \\
\text { inundado }\end{array}$ & $\begin{array}{l}\text { Vegetación } \\
\text { lacustre }\end{array}$ & $\begin{array}{c}\text { Vegetación } \\
\text { Costera }\end{array}$ & $\begin{array}{l}\text { Actividad } \\
\text { antrópica }\end{array}$ & $\begin{array}{c}\text { Cuerpo de } \\
\text { agua }\end{array}$ \\
\hline Bosque nuboso & 4677.5 & 0.1 & 0.0 & 0.0 & 0.0 & 0.0 & 9.4 \\
\hline Bosque & 158.4 & 35008.0 & 134.0 & 0.0 & 56.0 & 706.4 & 244.8 \\
\hline Vegetación lacustre & 0.0 & 0.0 & 7.6 & 384.4 & 0.0 & 0.0 & 8.3 \\
\hline Bosque inundado & 0.0 & 45.0 & 528.0 & 31.3 & 0.0 & 0.0 & 0.1 \\
\hline Manglar & 0.0 & 52.0 & 0.0 & 0.0 & 129.1 & 0.0 & 2.4 \\
\hline Actividad antrópica & 0.0 & 36.0 & 0.0 & 0.0 & 0.0 & 41.7 & 1.3 \\
\hline Cuerpo de agua & 0.0 & 38.0 & 0.1 & 16.7 & 0.5 & 0.9 & 17.9 \\
\hline
\end{tabular}

Cambio de uso año 1976-97 (ha)

\begin{tabular}{lccccccc}
\multicolumn{1}{c}{ Cobertura 1976 } & Bosque nuboso & Bosque & $\begin{array}{c}\text { Bosque } \\
\text { inundado }\end{array}$ & $\begin{array}{c}\text { Vegetación } \\
\text { lacustre }\end{array}$ & $\begin{array}{c}\text { Vegetación } \\
\text { Costera }\end{array}$ & $\begin{array}{c}\text { actividad } \\
\text { antrópica }\end{array}$ & $\begin{array}{c}\text { Cuerpo de } \\
\text { agua }\end{array}$ \\
Bosque nuboso & 4234.5 & 601.3 & 0.0 & 0.0 & 0.0 & 0.0 & 0.0 \\
Bosque & 1477.7 & 33585.0 & 62.0 & 0.0 & 27.0 & 23.0 & 6.0 \\
Vegetación lacustre & 0.0 & 0.0 & 44.2 & 367.3 & 0.0 & 0.0 & 20.9 \\
Bosque inundado & 0.0 & 82.0 & 581.3 & 7.0 & 0.0 & 0.0 & 0.0 \\
Vegetación costera & 0.0 & 75.0 & 0.0 & 0.0 & 109.6 & 0.0 & 1.2 \\
Actividad antrópica & 0.0 & 738.0 & 0.0 & 0.0 & 0.0 & 7.6 & 0.0 \\
Cuerpo de agua & 74.7 & 193.0 & 0.5 & 10.1 & 1.3 & 0.0 & 5.1 \\
\hline
\end{tabular}

Cambio de uso año 1997-2014 (ha)

\begin{tabular}{lccccccc}
\multicolumn{1}{c}{ Cobertura 1997 } & Bosque nuboso & Bosque & $\begin{array}{c}\text { Cobertura 2014 } \\
\text { inundado }\end{array}$ & $\begin{array}{c}\text { Vegetación } \\
\text { lacustre }\end{array}$ & $\begin{array}{c}\text { Vegetación } \\
\text { Costera }\end{array}$ & $\begin{array}{c}\text { Actividad } \\
\text { Antrópica }\end{array}$ & $\begin{array}{c}\text { Cuerpo de } \\
\text { agua }\end{array}$ \\
Bosque nuboso & 5717.8 & 69.1 & 0.0 & 0.0 & 0.0 & 0.0 & 0.0 \\
Bosque & 3260.3 & 31668.1 & 165.8 & 4.0 & 60.0 & 0.0 & 116.0 \\
Vegetación lacustre & 0.0 & 0.0 & 9.0 & 369.3 & 0.0 & 0.0 & 6.0 \\
Bosque inundado & 0.0 & 47.5 & 550.2 & 87.5 & 0.0 & 0.0 & 2.4 \\
Vegetación costera & 0.0 & 12.3 & 0.0 & 0.0 & 109.5 & 0.0 & 16.1 \\
Actividad antrópica & 0.0 & 27.8 & 0.0 & 0.0 & 0.0 & 3.1 & 0.0 \\
Cuerpo de agua & 0.0 & 2.2 & 0.2 & 18.1 & 0.9 & 0.0 & 11.9 \\
\hline
\end{tabular}

de 1477 ha y en 1997-2014 de 3260 ha, proceso contrario al descrito por Pounds, Fogden y Campbell (1999), ocurrido en Monteverde, donde las nubes han aumentado su altitud, lo que provoco el incremento de la temperatura superficial y con ello la reducción de este tipo de bosque.
La regeneración del área boscosa, especialmente en los sectores planos, fue un proceso rápido como plantea Bognounou et al. (2011), así como del resto de la península, pudo provocar un decrecimiento de la temperatura, facilitando que la presencia de las nubes sobre los cerros aumente, expandiendo el bosque nuboso en un 52 \% de 1997 a 2014. 
Sin embargo, esta información está influenciada por condiciones atmosféricas del momento de la toma de fotografía o imagen de satélite, debido a que las de 1970, 1976, 1997 se obtuvieron en los meses de enero y febrero cuando las precipitaciones eran menores y por lo tanto, también, la humedad, mientras la imagen de 2014 corresponde a agosto, que es uno de los meses de mayor volumen de precipitación y con mayor nubosidad, lo cual fue uno de los criterios utilizados para identificar los bosques nubosos. El número de fragmentos de esta categoría fue cinco en 1960 y 2014, 12 en 1976 y 1997, lo que refleja el proceso de conectividad funcional que experimenta el bosque nuboso en el área de estudio.

En el caso del bosque inundado se presenta un crecimiento de su superficie en un $11 \%$ durante el periodo 1960-1976, mientras que durante 1976-1997 el aumento fue $2 \%$ y $6 \%$ en 1997-2014. El incremento del periodo 1997-2014 se puede justificar, como se mencionó anteriormente, por las condiciones del momento en que fue tomada (agosto) la imagen, cuando se presenta una alta precipitación y con ello se eleva el nivel de agua de los ríos. La deforestación en el área de estudio, aunque fue escasa, se localizaba en un lugar estratégico como es alrededor de la planicie aluvial que forma los ríos Sirena y Claro, que son los drenajes principales de la cuenca hidrográfica, que estaban habitados por bosques y vegetación lacustre. Así, en el periodo 1960-1976, se presenta un acelerado proceso de expansión de la frontera agrícola. Asimismo, 134 ha de bosque se transformaron en bosque inundado y 7.6 ha de vegetación lacustre, mientras en 1976-1997 el bosque se expandió bajo la reducción de 62 ha de bosque inundando y 44 ha de vegetación lacustre. Para el lapso 1997-2014, 165 ha de bosque y 9 ha de vegetación lacustre se convierten en bosque inundado; los cuales se recuperan por los procesos de regeneración natural cuando se detiene la actividad antrópica, e influenciados por los procesos de escorrentía que son más lentos en los bosques, lo que incrementa el nivel del agua y facilita la expansión de este tipo de vegetación.
Asimismo, se identificó que las diversas categorías de vegetación coexisten, generando dinámicas de vecindad específicas que están determinadas por factores particulares. Por ejemplo, existe una coexistencia entre el bosque y el bosque inundado, que provoca que su superficie se expanda y contraiga en función de la distribución y volumen de precipitación. El bosque inundado, dominado por la palma de yolillo (Raphia taedigera), que se confina alrededor de la laguna Sirena, representó entre el $1.4 \%$ y el $1.7 \%$ del área de estudio, con una tendencia a incrementarse. Relacionada con el bosque inundado se encuentra la vegetación lacustre, la cual ocupa principalmente el espejo de agua de la laguna del río Sirena, con un área de 0.9 y el $1.1 \%$ de la superficie del Parque. Estas dos categorías se encuentran íntimamente ligadas e igualmente afectadas por el volumen y la distribución de la precipitación que recibe anualmente la cuenca del río Sirena.

En el caso de la vegetación costera, localizada en la desembocadura de los principales ríos, representa entre el 0.4 y $0.3 \%$ de la superficie, presentando reducciones mayores en área, similar a lo encontrado por Acuña (2016) en el delta de río Térraba, quien concluyó que durante los periodos de 1948-1972, así como en 1972-1992, los procesos erosivos son el principal factor que provoca la pérdida del manglar, lo cual puede estar ligado con las dinámicas del cambio climático.

Las transformaciones ambientales de la actualidad obligan a comprender las dinámicas espaciales de la vegetación con el objetivo de apoyar su conservación. De acuerdo con los resultados, el establecimiento del Parque Nacional Corcovado en 1975, redujo el proceso de desforestación y generó condiciones para la restauración de los espacios impactados, por ejemplo, 706 ha de bosque fueron convertidas en espacios agropecuarios en el periodo 19601976, mientras que en 1976-1997 fueron 23 ha y en 1997-2014 este proceso se detuvo. Lo anterior evidencia lo apropiado del momento de establecimiento del Parque, lo cual permitió preservar y restaurar los ecosistemas que urgían de su conservación. 
Las categorías de vegetación identificadas para esta investigación, según el trabajo de campo y los diferentes resultados, evidencian la interrelación entre las mismas que provoca que se expandan y reduzcan en función de factores como el volumen y distribución de precipitaciones; así como la presencia temporal de nubosidad y la recuperación de la cobertura boscosa, tanto dentro de los límites del parque como dentro de la misma Península de Osa. La relación de interdependencia se identificó entre el bosque y el bosque nuboso, del mismo modo entre el bosque y el bosque inundado. Basado en la necesidad de continuar monitoreando la distribución espacio-temporal de la vegetación del Parque Nacional Corcovado, tanto a largo como a corto plazo, es recomendable establecer parcelas permanentes en diferentes sitios que permitan evaluar la riqueza y evolución de los diferentes tipos de vegetación, para implementar acciones que favorezcan la preservación de los ecosistemas que conserva el Parque, especialmente para profundizar con la interdependencia entre las categorías de vegetación identificadas.

\section{AGRADECIMIENTOS}

A los colegas guardaparques del Parque Nacional Corcovado por la ayuda incondicional en la colecta de datos durante todos estos años. A la Vicerrectoría de Investigación de la Universidad Nacional por el apoyo brindado.

\section{RESUMEN}

Los estudios a nivel regional que evalúan las dinámicas espacio-temporales de la vegetación en Costa Rica, especialmente, dentro de los Parques Nacionales son escasos. Así, considerando aportar en este vacío de conocimiento, este artículo analiza la distribución espacio-temporal de la vegetación dentro del periodo 1960-1976, 1992, 1997 y 2012 en Parque Nacional Corcovado, localizado en la Península de Osa y catalogado como el bosque tropical lluvioso más septentrional en la costa pacífica de América. Además, esta área contiene una riqueza de biodiversidad, fundamentada en su antigüedad geológica, el aislamiento que presentó durante largos periodos; así como las condiciones climáticas particulares que generan ecosistemas únicos como bosques nubosos relacionados con brisa marina a alturas de más de $500 \mathrm{msnm}$. Este estudio evalúa la distribución espacial de la vegetación a partir de mapas resultantes del proceso de fotointerpretación de imágenes del 1960, 1976, 1997 y 2012, así como del análisis del índice de paisaje. Se concluye que las transformaciones espacio-temporales de la vegetación durante el periodo de estudio han sido mínimas, y el hecho de que hayan sido escasas las áreas impactadas por la actividad antrópica, generó una restauración ecológica importante durante las últimas décadas. Se encontró una relación de expansión y contracción entre el bosque nuboso y bosque, así como este último y el bosque inundado, en función de la recuperación de la cobertura boscosa dentro del Parque Nacional y de la Península de Osa, y el volumen y distribución de la precipitación. Asimismo, este estudio propone la necesidad de establecer el monitoreo permanente de la vegetación para esclarecer las relaciones que se establecen entre estos tipos de vegetación.

Palabras clave: Bosques tropicales lluviosos; cartografía de vegetación; Parque Nacional Corcovado; monitoreo de vegetación; categorías de vegetación

\section{REFERENCIAS}

Acuña, F. (2016). Análisis espacio-temporal de la evolución geomorfológica, los cambios en los usos y coberturas de la tierra, asi como la distribución del mangle en el humedal nacional Térraba-Sierpe en el periodo de 1948 - 2012. (Tesis.) Escuela de Ciencias Geográficas, UNA. Costa Rica.

Aldana, A., \& Bosque, J. (2008). Cambios ocurridos en la cobertura/uso de la tierra del Parque Nacional Sierra de la Culata. Mérida-Venezuela. Período 1988 - 2003. GeoFocus. Revista Internacional de Ciencia y Tecnología de la Información Geográfica, 8, 139-168.

Bognounou, F., Morton, R., Ayangma, S., Laurence, J., Bjorkman, C., Bylund, H., ... Oden P. (2011). Stand structure of monocotyledons and Dicotyledons in differents successional stage in Corcovado Nacional Parks, Costa Rica. Bois et forêts des tropiques, 307(1), 33-40.

Calvo, A., \& Ortiz, E. (2011). Fragmentación de la cobertura forestal en Costa Rica durante los periodos 1997 2000 y 2000-2005. Revista Forestal Mesoamericana Kurú, 9(22), 10-21.

Calvo, J., Watson V., Bolaños, R., Quesada C., Sánchez, A, Gonzáles, P., \& Ramírez, J. (1999). Estudio de la cobertura forestal de Costa Rica empleando imágenes Landsat 1986/1987 y 1996/1997. XI Congreso Agronómico Nacional y de Recursos Naturales. San José, Costa Rica.

Carrillo E. (2000). Ecology and conservation White-lipped peccaries and jaguars in Corcovado National Park, 
Costa Rica. (Tesis doctoral). Universidad de Massachusetts, Estados Unidos.

Chuvieco, E. (2010). Teledetección ambiental. La observación de la Tierras desde el espacio. España: Editorial Arial.

Denyer, P., \& Kussmaul, S. (2000). Geología de Costa Rica. Cartago, Costa Rica: Editorial Tecnológica de Costa Rica.

Domínguez, J. A. (2011). Transformación físico-espacial de los paisajes boscosos del sector sur del Parque Nacional Natural los Katíos (1989-2010). Perspectiva Geográfica: Revista del Programa de Estudios de Posgrado en Geografia, 16(1), 31-54.

Flores, D., Suvires, G., \& Dalmasso, A. (2015). Distribución de la vegetación nativa en ambientes geomorfológicos cuaternarios del Monte Árido Central de Argentina. Revista Mexicana de Biodiversidad, 86(1), 72-79. doi: 10.7550/rmb.40248

Foster, D. R., Orwlg, D. A., \& McLachlan, J. S. (1996). Ecological and conservation insights from reconstructive studies of temperate old-growth forests. Trends in Ecology \& Evolution, 11(10), 419-424.

Geist, H., McConnell, W., Lambin, E. F., Moran, E., Alves, D., \& Rudel, T. (2006). Causes and trajectories of land-use/cover change. Land-use and land-cover change (pp. 41-70). Germany: Springer Berlin Heidelberg.

Gómez, L. D. (1986). Vegetación de Costa Rica: Apuntes para una biogeografía costarricense. Capítulo IX. Los tipos de Vegetación de Costa Rica. Vegetación y clima de Costa Rica. V:1. Costa Rica: EUNED.

Green, G. M., Schweik, C., \& Randolph, J. C. (2005). Linking disciplines across space and time: Useful concepts and approaches for land-cover change studies. In E. F., Morand \& E., Ostrim (Eds.) Seeing the forest and the trees (pp. 61-80). Cambridge, MA: MIT Press.

Hammel, B. E., Grayum, M. H., \& Aguilar, R. (2014). Tapirira lepidota (Anacardiaceae), a unique new species from the Golfo Dulce region of Costa Rica. Phytoneuron, 10, 1-5.

Hilje, L. (2013). Trópico agreste: Las huellas de los naturalistas alemanes en la Costa Rica del Siglo XIX. Instituto Tecnológico de Costa Rica. Cartago,Costa Rica: Editorial Tecnológica.

Kappele, M., Castro, M., Acevedo, H., González, L., \& Monge H. (2002). Ecosistemas del área de Conservación Osa (ACOSA). Santo Domingo, Costa Rica: INBIO.

Lewis, B. (1983). Reseña histórica de la población y los recursos naturales de la Península de Osa, Pacífico
Sur, 1848-1981. Revista Geográfica de América Central, 2(17-18), 123-130.

Manies, K. L., \& Mladenoff, D. J. (2000). Testing methods to produce landscape-scale presettlement vegetation maps from the US public land survey records. Landscape Ecology, 15(8), 741-754.

Morera, C. (2001). Ecoturismo y desarrollo local en la Península de Osa. Revista Geográfica de América Central, 39, 41-74.

Morera, C. (2010). Estructura del Paisaje y Desarrollo Turístico en la Fila Brunqueña, Costa Rica. Revista Geográfica de América Central, 44, 91-108.

Morera, C. (2015). Ordenamiento territorial: desafíos para una sociedad en crisis ecológica. GEOUSP: Espaço e Tempo (Online), 19(2), 312-323. doi:10.11606/ issn.2179-0892.geousp.2015.102804

Morera, C., \& Sandoval, L. (2012). Estructura del paisaje y desarrollo turístico en Cahuita, Talamanca. Revista Geográfica de América Central, 48E, 169-196.

Morera, C., \& Sandoval, L. (2013). Los procesos de fragmentación y la conectividad forestal en Costa Rica: Dos casos de estudios. In C. Morera. Geografia, Paisaje y Conservación. Heredia, Costa Rica: ECG - UNA.

Morera, C., \& Sandoval, L. (2015). Aplicabilidad de indicadores de estructura de paisaje para evaluar transformaciones en escenarios tropicales. Métodos y Técnicas Geográficas Aplicadas al Ambiente: Una Visión Latinoamericana. Universidad Autónoma de San Luis Potosí, México: EUNA (en prensa).

Nagendra, H., Southworth, J., \& Tucker, C. (2003). Accessibility as a determinant of landscape transformation in western Honduras: linking pattern and process. Landscape Ecology, 18(2), 141-158.

Palacio-Aponte, G. (2014). Geoindicador de modificación de la cobertura aplicado a un ambiente costero tropical. Revista de Biología Tropical, 62(3), 1111-1128.

Pounds, J. A., Fogden, M. P., \& Campbell, J. H. (1999). Biological response to climate change on a tropical mountain. Nature, 398(6728), 611-615.

Quesada, F., Jiménez, Q., Zamora, N., Aguilar, R., \& González, J. (1999). Árboles de la Península de Osa. Santo Domingo, Heredia, Costa Rica: INBIO.

Sahagún, F. J., Reyes, H., Flores, J. L. F., \& Vargas, L. C. (2011). Modelización de escenarios de cambio potencial en la vegetación y el uso de suelo en la Sierra Madre Oriental de San Luis Potosí, México. Journal of Latin American Geography, 10(2), 65-86.

Sánchez, A., Benoit, R., Calvo, J., \& Moorthy, I. (2002). Dynamics of Tropical deforestation around National Parks: remote sensing of forest change on the Osa 
Peninsula of Costa Rica. Mountain Research and Development, 22(4), 352- 358.

Sánchez, A., Foley, S., Hamilton, S., Calvo, C., Arroyo, P., \& Jiménez, V. (2002). Estudio de Cobertura Forestal de Costa Rica imágenes Landsat TM/ para el año 2000. San José, Costa Rica: Centro Científico Tropical.

Santamaría-Aguilar R., \& Lagomarsino, L. P. (2015). Synopsis of Couepia (Chrysobalanaceae) in Costa Rica, with a description of two new species. Phytota$x a, 233(1), 69-79$.

SINAC. (1996). GRUAS I: Propuesta de ordenamiento territorial para la Conservación de la Biodiversidad en Costa Rica. Mimeografiado San José, Costa Rica.

SINAC. (2007). GRUAS II: Propuesta de ordenamiento territorial para la Conservación de la Biodiversidad en Costa Rica. Mimeografiado San José, Costa Rica.

Vargas, G. (2009). La vegetación de Costa Rica. Un estudio geográfico y ecológico. San Pedro, Costa Rica: Librería Alma Mater.
Vaughan, C. (1981). Parque Nacional Corcovado: Plan de manejo y desarrollo. Heredia, Costa Rica: EUNA.

Velázquez, A., Durán, E., Ramírez, I., Mas, J. F., Bocco, G., Ramírez, G., \& Palacio, J. L. (2003). Land use-cover change processes in highly biodiverse areas: the case of Oaxaca, Mexico. Global Environmental Change, 13(3), 175-184.

Veldkamp, A., \& Fresco, L. O. (1996). CLUE-CR: an integrated multi-scale model to simulate land use change scenarios in Costa Rica. Ecological modelling, 91(1), 231-248.

Willie, A. (1987). Corcovado meditaciones de un biólogo: Un estudio ecológico. San José, Costa Rica: EUNED.

Young, K. R. (2014). Biogeography of Anthropocene: novel species assemblages. Progress in Physical Geography, 38(5), 664-673.

Zamora, N. (2013). Una nueva especie de Schnella (Leguminosae, Caesalpinioideae: Cercideae) para Costa Rica. Phytoneuron, 12, 1-6. 